\title{
La dimensión sacramental de la Iglesia según San Agustín
}

Si es cierto que el tema de la Iglesia interesó a los teólogos de una manera peculiar en las últimas décadas ${ }^{1}$, no lo es menos que, a raíz del Concilio Vaticano II el empeño por penetrar y profundizar en el rico e inagotable contenido de su misterio se ha acentuado de tal manera que bien puede calificarse de sorprendente. En nuestros mismos días estamos asistiendo a una proliferación de ensayos en torno al tema como jamás se había conocido en la historia de la teología ${ }^{2}$.

1 Ya en el esquema del Concilio Vaticano $\mathrm{I}$, que no pudo ser, por circunstancias conocidas, discutido ni aprobado, se perfilaban los frutios de las nuevas tendencias y se apreciaba el progreso de la doctrina eclesiológica, presentándose esquemas de una doctrina más completa y de más amplias aplicaciones, tanto por lo que se refiere a la naturaleza de la Iglesia como por lo que se refiere a sus propiedades y sus poderes. Aunquie aquella doctrina, por fuerza de las circunstancias, quedó en proyecto casi en su totalidad, sin embargo tuvo influencias posteriores decisivas, incluso en el Magisterio de la Iglesia, como en las Encíclicas Satis Cognitum y Mystici Corporis, de Løón XIII y PÍo XII respectivamente. Esto supuso una razón más a favor de las nuevas tendencias que se perfilaban por entonces y que ofrecían indudable interés, sobre todo, por la: reflexiones sobre la esencia de la Iglesia como sociedad y comunidad, sobre su forma jurídica y sobre su realidad interior, la caridad, la obra del Espíritu Santo como alma de la Iglesia. Pero el aspecto nuevo de más relieve, que luego cristalizará en doctrina del Magisterio, fue el de la consideración de la Iglesia como "Cuerpo Místico de Cristo". En este movimiento con matices tan concretos y acentuados -que se había iniciado anteriormente con el romanticismo alemán- no deben pasarse por alto nombres como J. A. MöHLER, J. Franzelin, J. KLEUTEN, M. J. SChEEBEN, que abrieron las puertas a nuevas orientaciones. Cfr. I. BACKEs, "Gottes Volk im Neuen Bunde": Trierer Theol. Zeitschrift 70 (1961) 80 ss; ; F. HOLBOCH - TH. SARTORY, El misterio de la Iglesia. Fundamentos para una Eclesiología, Barcelona 1966, 283 ss.

2 Hoy, después de la importancia que el Vaticano II dio a la Iglesia, es evidente que los estudios siguen en plena floración, y som muchos los aspect'os que merecen un estudio más detallado y sereno para llegar, en toda la doctrina que se nos ofrece como perteneciente al depósito sagrado de la Iglesia, a las últimas conclusiones. Como muestra, y en estle sentido, podemos citar las obras siguientes: La Iglesia del Vaticano II. Estudios en torno a la Constitución conciliar sobre la Iglesia. (Obra colectiva en dos volúmenes, dirigida por G. BARAunA) (Barcelona, J. Flors, 1966); Concilio Vaticano II. Comentarios a la Constitución sobre la Iglesia (Madrid, BAC 1966); La Iglesia del Concilio Vaticano II (Estudios de J. Nicolau, J. Daniélou, P. Molinari, Narciso G. Garcés) (Bilbao, El Mensajero, 
Al Concilio Vaticano II llega ya una doctrina eclesiológica en plena cbullición y en algunos aspectos madura. El Concilio ha recogido estas orientaciones nuevas de la teología y las ha hecho suyas, juntamente con otras todavía no definitivas, a fin de que vaya enriqueciéndose el depósito de la revelación con la savia vivificante y renovada.

De esta forma se ha abandonado lo que han dado en llamar algunos la visión estática de la Iglesia para entrar de lleno en la consideración C: $\in$ las propiedades ónticas de la visión dinámica o dimensión del misterio sobrenatural de la misma ${ }^{3}$. De ahí que sean los aspectos cristocéntrico, pneumatológico, sacramental, y escatológico de la Iglesia los que resumen hoy las perspectivas de las reflexiones teológicas ${ }^{4}$.

Más que hablar del abandono de unos conceptos jurídicos que tienen su razón y su función inalienable, habrá que hablar del enriquecimiento, del complemento y la perfección de los mismos, al ser considerados desde otras perspectivas necesariamente conexas, pero de óptica más completa. Planteadas así las cosas tampoco hay por qué extrañarse

1966), etc. Merece especial atención la primera, puesto que se trata de una obra en la que colaboran los más destacados especialistas internacionales y en los esitudios que en ella se nos ofrecen se tocan prácticamente todos los aspectos de la eclesiología según la doctrina elaborada por el Concilio: hasta dónde se llegó, tendencias y límites de una doctrina que si no puede considerarse como nueva, sin embargo está siendo estudiada bajo nuevas y prometedoras perspectivas. Eu este sentido tampoco podemos olvidarnos de las apontaciones bibliográficas de BLUET y BOUYER, interesantes por la abundancia de autores que señalan en torno al itema de la Iglesia como misterio. Podemos decir que casi es una excepción la revista en la que no se habla o trata algún aspecto de la Iglesia según el Vaticano, en números dedicados expresamente o en trabajos sueltos, v. gr. Selecciones de Teología, Angelicum, Gregorianum, Concilium, etc.

3" Cfr. J. L. WITTE, "La Iglesia, "Sacramentum unitatis" del cosmos y del género humano": La Iglesia del Vaticano. II, Barcelona 1966, 505-535. En realidad son muchos los autores que reinciden en estas afirmaciones e insinuaciones. Y Congar, por ejemplo, constata el hecho y lo explica en estos términos: "La renovación de la idea de la Iglesia en la época moderna se ha hecho merced a un descubrimiento de su dimensión del misterio sobrenatural, más allá de la presentación demasiado exclusiva del engranaje jerárquico, al cual se ceñía con frecuencia la eclesiología polémica nacida de la Contrarreforma..." ConGAR, "Dogma cristológico y eclesiología. Verdad y límites de un paralelo": Santa Iglesia, Estela, Barcelona 1965, 90 ss. Vuelve sobre este mismo concepto en otras parties, v. gr., 398 ss. La bibliografía en los últimos años se multiplicó de tal forma que resulta prácticamente imposible abarcarla toda. Basta recordar aquí la síntesis del P. Y. CoNGAR, "Crónica de treinta años de estudios eclesiológicos": Santa Iglesia, y la del P. Domínguez DEL VAL, "La Eclesiología en los últimos años (1950-1964). Orientaciones bibliográficas": Salmanticensis 12 (1905) 319-394. Estas obras nos dan una idea aproximada de lo que era la bibliografía eclesiológica antes diel Concilio.

4 Pueden consultarse en relación con estas nuevas perspectivas: J. WoDKA, Mysterium Kirche I, 470-471 (GE.Schichte des Kirchenbegriffs); Y. M. CoNGAR, Kirche: Dogmen- geschichtlich en TRIEs, Handbuch theologischer Grundbergrif (München 1962) I, 801-812. Igualmente la bibliografía señalada arriba. 
de cierta terminología que de otra manera pudiera parecer exagerada o incluso pedante ${ }^{5}$.

Es significativo, e interesa hacerlo notar, que todas estas nuevas concepciones eclesiológicas se están efectuando bajo un lema que está haciendo gala, reiteradamente, de fidelidad a una época lejana pero muy rica en contenido y dimensiones teológicas: la patrística. La vuelta a los Padres, que tantos caminos ha abierto siempre en la teología, ha sido sin duda un determinante decisivo en estas nuevas orientaciones. Ahora bien, esta mirada hacia dentro - misterio de una realidad celeste y terrestre, eterna y temporal, visible e invisible- y hacia el pasado - patrística - , tiene un sentido que la hace transcender el mero ámbito histórico y que podría concretarse, como se ha escrito, en triple ejercicio de reflexión, acción y pasión ${ }^{6}$.

Es decir, que si hoy se está volviendo a aquéllo no es únicamente para recordar, representar lo que ya presentaron los Padres, sino más bien para seguir buceando, inmersos en aquel fondo, y profundizar en lo que jamás podrá ser agotado por el limitado entendimiento humano, puesto que se trata de un misterio ${ }^{7}$. Pero sí para enfrentarse al misterio

5 Es lo que expresaba Pablo VI con su mesurada palabra: "Nos parece que ha llegado el tiempo en que se deba explorar, invesittigar y exponer cada vez más la verdad sobre la Iglesia, acaso no con aquellas enunciaciones' solemnes que llaman definiciones dogmáticas, sino más bien empleando declaraciones con las que la Iglesia con una forma de enseñanza más grave y más clara, se declara a sí misma qué es lo que de sí misma siente (Alocución al Concilio, 29 sept. 1963). Cfr. Congar, "Apliciacciones: noción organicista de la Iglesia. Idea de "encarnación continuada": o. c., 89 s.s.; O. G. HERNÁNDEZ, "La nueva conciencia de la Iglesia y sus presupuesto histórico-teológicos": La Iglesia del Vaticano II, Barcelona 1966, 249.

6 "La Iglesia progresa en el conocimiento de la revelación, y, consiguientemente en el conocimiento de sí misma por un estudio renovado siempre de sus fuentes propias (la Biblia, Liturgia, Padres), explicitando mediante un trabajo discursivo las implicaciones de las verdades reveladas; por un conocimiento experimental de connaturalidad, fruto de una confirmación de la propia vida con el misterio; no sólo pues de un estudio de la Iglesia, sino de una "pasión" de Igle sia; es en el orden religioso "el parecer" (Tà Ceia pacein) el conocimiento más reveliador”, O. G. HERNÁNDEZ, o. c., 256-257.

Es una vivencia nueva de la Iglesia que responde a unas exigencias temporales y dimensionales, cronológicas y circunstanciales hodiernas, como en la época patrística respondió a necessidades de urgencia también contemporáneas, v. gr. en Cipriano, Agustín, etc. Mientras que más tarde, en el siglo XIV, surgía la necesidad concreta de la defensa diel Papado, y por lo mismo se cargaba el acento casi exclusivamente en los elementos externos, sobre todo, en la jerarquía. Como muestra puedien citarse los primeros tratados sistemáticos sobre la Iglesia de SANTIAGO DE VITERBo, De regimine christiano ad Clementem $V$, libri auatuor (130); EgIDIo Romano. De potestate ecclesiastica; AgUsTín TRIUNFo, Summa de potestate ecclesiastica (1328).

7 Es uno de los aspectos en que últimamente más se ha insistido. Tal vez sea de Lubac quien más ha profundizado y ell que mejor ha señalado la amplitud y los límites en su conocido libro: Méditation sur l'Eglise (AUBIER, Editions 
con la humildad suficiente, como nos recuerda $H$. de Lubac, para profundizar en las relaciones del misterio con Dios, Cristo y los sacramentos. Por tanto no se trata de doctrina nueva, absolutamente hablando, sino de doctrina que va surgiendo al ritmo de los tiempos del caudal inagotable de la tradición escriturística, litúrgica y patrística.

Por otra parte, llegar a concretar hasta qué punto se encuentran ya en los Padres estas nuevas perspectivas así como el alcance de una terminología nueva y su relación con la antigua, es tarea complicada y nada fácil, aunque sí puede afirmarse que más que tratarse de una doctrina nueva se trata de una perspectiva nueva, de un proceso lógico dentro del desarrollo doctrinal eclesiológico ${ }^{8}$.

\section{DiMENSIÓN SACRAMENTAL.}

Lo que decimos en general de estos aspectos nuevos debemos decirlo en concreto de la sacramentalidad, y lo que decimos en general de los Padres podemos decirlo en concreto de San Agustín, quien, por otra parte, como es sabido, contiene una riqueza inmensa doctrinal en torno a la Iglesia, de tal manera que en él "se debe buscar la fuente primera y común de todos los tratados sobre la Iglesia" (Grabowski).

Es claro, sin embargo, que no podemos hacernos la ilusión de encontrar en él una terminología explícita sobre la dimensión sacramental de la Iglesia, pero tampoco significa esto que no nos proporcione abundantes consideraciones que el mismo Concilio y la teología postconciliar está poniendo en evidencia ${ }^{9}$. No podemos buscar ésto en

Montagne, Paris 1954). En estie mismo sentido podemos recordar igualmente a Congar, E'squise du mystère de L'Eglise, Paris 1941, 26; MERsch, "Corp Mystique et Spiritualité": Dict. de Spirit. II, col. 2.378; HoLbock, Der eucharistiche und der mystiche Leib Christi, Roma 1941.

8 La índole de esstie progreso es afirmada con frecuencia por autores diversos de nuestros días implicadois en esta temática. Por ejemplo: "Progreso no tanto por análisis cuanto por síntesisis. Su labor radica no tanto en las orientaciones aisladas cuanto en la visión orgánica total de todo el misterio que ha intentado la Iglesia; en el esfuerzo hecho por descubrir su lugar propio dentro del horizonte general de la historia de la salvación y de la historia humana total; en el intento de distinguir qué elementios le son esenciales y cuáles accidentales, mutables e inmutables; de colocar en el centro de su conciencia lo que es el centro de su ser y en el corazón de su esfuerzo lo que es el corrazón de su misión", HERNÁNDEZ, o. c.. 260. Otros, por su partie, hablan de la "nueva concepción eclesiológrica", Cfr. SMulders, "La Iglesia como sacramento de salvación": La Iglesia del Vaticano II, Edit. Juan Flors, Barcelona 1966, 377 .s.

9) El Vaticano II afirma la sacramentalidad de la Iglesia en diversas ocasiones. Es la primera vez que en un documento oficial, en una constitución se nos habla de la Iglesia como sacramento. Así por ejemplo: "Cum autem Ecclesia sit in Christo veluti sacramentum seu signum et instrumentum intimae cum Den 
Agustín por muchas razones, pero baste aquí señalar la dificultad misma del tema que hace que ni siquiera hoy sea doctrina clara ni unánime en los mismos teólogos de nuestro tiempo que se ocupan de poner de relieve este aspecto ${ }^{10}$.

Pero no por eso deja de tener sumo interés este aspecto sacramental de la Iglesia según San Agustín. Precisamente una de las lagunas que, debido a la orientación que han tomado los estudios eclesiológicos, se hace cada día más notoria en la extraordinaria obra de Grabowski, La Iglesia. Introducción a la Teología de San Agustín, es la referente al tema de las relaciones entre sacramento e Iglesia, o más concretamente llamado la sacramentalidad de la Iglesia ${ }^{11}$. Y ello es más evidente porque resulta un hecho palpable que Agustín abunda en doctrina lo mismo sobre la Iglesia que sobre los sacramentos ${ }^{12}$.

Por otra parte, si bien Grabowski no ha afrontado directamente este tema, tiene, sin embargo, unas palabras muy significativas que nos indican que no es en modo alguno ajeno a San Agustín, y que, incluso, señalan las perspectivas y posibilidades de esta doctrina agustiniana: "San Agustín no aplica el término "sacramento" a la totalidad de la

unionis totius generis humani unitatis'" (Lumen Gentium, n. 1, BAC, 9); "Deus congregationem eorum qui in Iesum..." (Ibid. 24); "Corpus suum quod est Ecclesia..." (Ibid., 93). Sabemos por lasł Actas del Concilio que la oposición venía sobre todo por el temor o peligro que algunos Padres veían para el pueblo y gentes menos instruídais no acostumbradas a distinciones teológicas. Cfr. DANIELOU, o. c., 47.

10 Y. CoNGAR llama la atención en un comentario al libro de SEMMELRoth, Die kirche als Ursackrament (Francfort 1953), trad. castellana, La Iglesia como sacramento original (San Sebastián, Dinor 1963). Comentario en RSPT (1953), t. 33, 752; incluído en Saint Eglise (Paris, Cerf. 1963), 623 trad. castellana, Santa Iglesia (Barcelona, Estela 1965) 550-551. Del mismo autor: "Die 23-39. Igualmente del mismo autor": "Um die Einheit des Kirchenbegriffes": Kirche als sichtbare Gestalt der unsichtbaren Gnade": Scholastik (1953), t. 28, Fragen der Theologie heute (Eisiedeln 1957) 319-337, trad. castellana, Panorama de la teología actual (Madrid, Guadarrama 1961);' G. HowrmanN, "Die Kontroverse über den Kirchenbegriff": Trierer theol. Zeitsch (1956) 109-114, 174-182; HoMer, L'Eglise est une comunion (Paris, Cerf. 1965), trad. castillana, La Iglesia es una comunión (Barcelona, Estela 1965) 84-85.

11 El P. ORoz en la Presentación que hace a la obra se lamenta con estas palabmas: "En el índice o esquema del libro de Grabowski notamos la ausencia de algún que otro tema que actualmente interesa en un trabajo de Eclesiología. Nos queremos ref'erir principalmente al ecumenismo, a la Iglesia-Sacramento... Es cierto que Grabowski alude al papel de los sacramentos en la constitución de la Iglesia, pero la sacramentalidad die éstia no aparece en plena luz...", Stanislaus J. GrabowsKI, La Iglesia. Introducción a la Teología de San Agustin, Madrid 1965, XXIX. Esto mismo lo repite en Augustinus, donde reproduce la Presentación que hace a la obra de Grabowski, "En torno a una eclesiología agustiniana": Augustinianus XI, 42-43 (1966) 269.

12 L. VilleTTE, Foi et Sacrament, vol. I: Du Nouveau Testament à Saint Augustin, Paris 1959. 
Iglesia. Pero quienes lo hicieron posteriormente concuerdan con su pensamiento" 13 .

El que no se trate este aspecto en esta obra, la más completa sobre la eclesiología agustianiana hasta hoy, resalta aún más por el sesgo y la orientación que han tomado en llos últimos tiempos tanto los estudios eclesiológicos como la agustinología.

La primera dificultad seria con que nos enfrentamos en el intento de comprender e interpretar a San Agustín, es la problemática que nos presenta el concepto y la terminología de la palabra "sacramento", ya que en Agustín, como se sabe, no tiene una significación única y ofrece, como en otros Padres, sentidos muy diversos.

Por otra parte, tampoco se puede hablar aquí de "sacramento" en sentido estricto, ya que como doctrina de fe se nos impone la absoluta certeza de que los sacramentos de la Nueva Ley son ni más ni menos que siete ${ }^{14}$.

Pero ni esa palabra tenía entonces ese significado único, estricto, ni lo había tenido en los Santos Padres y en la Sagrada Escritura. El Vaticano II aplica esta terminología a la Iglesia apoyándose justamente en el pensamiento de los Santos Padres y en la misma Escritura. No es que sea muy frecuente en los Santos Padres, como tampoco en el Nuevo Testamento, pero sí son característicos y significativos los lugares en que se encuentra esta expresión o su equivalente, v. gr. la Didache, Gregorio Niceno, Cipriano, Ambrosio. Por lo que se refiere a San Agustín la cuestión se centra en las palabras "misterio" y "sacramento". Esta última palabra es rara, aunque significativa. $\mathrm{Y}$ es precisamente el alcance de esta significación lo que nos interesa analizar.

El ámbito de este concepto sacramental es más amplio que el recogido por Trento, y explicado según la forma agustiniana: "Invisibilis gratiae visibilis forma" 15 , forma lapidaria, pero que no agota ni encierra en sí toda la amplitud que San Agustín dio a lo sacramental. ¿Cuál puede ser la dimensión de este concepto en la doctrina de San Agustín?

13. Stanislaus J. Grabowski, La Iglesia. Introducción a la Teología de San Aguistín, Madrid 1965, 171. No es nuestra pretensión afrontar en estas páginas toda la problemática que encierra el carácter de la sacramentalidad de la Iglesia en San Agustín. Esto exige sin duda un estudio más amplio que el que ahora intentamos. Nos limitamos únicamente a constaitar de manera muy elemental alguno de los aspectos doctrinales que se señalian hoy como base de la dimensión sacramental de la. Iglesia.

14 H. DENZINGER, Enchiridion Sympolorum Definitionum et Declarationum de rebus fidei et morum, Editio 24-25, 1948, Herder, Barcelona, n. 844, 300.

15 San Agustín, Quaest. in Hept. 3, 84, ML. 34, 712; Denz. 876, 305. 
La respuesta, como hemos dicho, exigiría un estudio muy detallado y más amplio que lo que ahora intentamos. De todas formas, dentro de estas ambiciones, puede ayudarnos a comprenderlo el sentido de este término en los primeros siglos y una rápida consideración a su doctrina.

Generalmente el término latino sacramentum es traducción del término griego musterion (Mc. 4, II), pero no agota su amplia significación aunque participe de ella. Término, por otra parte, de contenido muy diverso tanto en el sentido religioso como en el profano ${ }^{16}$.

En la Sagrada Escritura encontramos la palabra sacramento con un sentido muy amplio. Unas veces como misterio, verdad escondida y oculta, sin que por eso haya de entenderse en el sentido de algo oscuro $e$ ininteligible ${ }^{17}$. Otras veces equivale a un signo que de alguna manera produce lo que significa. Por ejemplo, se llama sacramento a la circuncisión de la Ley Antigua: en cuanto que era signo de la incorporación al Pueblo escogido de Dios y por ella se realizaba. En pasajes del Antiguo Testamento encontramos otros matices diversos, como, por ejemplo, cierta santificación personal ${ }^{18}$.

Es precisamente la relación entre estas dos palabras misterio y sacramento la que puede darnos la clave para una exacta comprensión del ámbito dimensional de la Iglesia como sacramento.

Interesa destacar en este sentido religioso la significación de "plan salvífico", de Dios respecto a la humanidad, plan que sólo es revelado a algunos escogidos ${ }^{19}$. Es una revelación que implica una lazo de amistad y de confianza por parte de Dios con los elegidos ${ }^{20}$.

La concepción paulina del misterio es de un interés máximo tanto por lo que en sí encierra cuanto por las consecuencias. El Apóstol hace uso de dicho vocablo no menos de veinte veces en sus cartas. $\mathrm{Y}$ aun cuando los especialistas no se han puesto de acuerdo en todos los matices al determinar el contenido de esa palabra en el Apóstol, sí han ilegado, sin embargo, a algunas conclusiones muy significativas: el

16 En este ámbito profano el significado suele ser: consejo secreto del rey y su plan de guerra: v. gr. en Judit $2,2$.

17 Sap. 2, 22; 6, 24; Dan. 2, 18 ss.; Mc. 4, 11; Col. 1, 26.

18 Conc. Florent. Dec. Pro Armenis. Denz. 695, 712.

19 Sap. 2, 22ú Dan. 2, 27, 45.

20. "La revelación del misiterio lleva consigo una relación de confianza, una consagración. Esta separa a los consagrados de los que están fuera, tal como aparece en los textos donde los sinópticos usan dicha palabra: "Porque a vosotros os ha sido dado conocer los misterios deil reino de los cielos, pero a ésos, no". SMULDERS, o. c., 382. 
misterio para San Pablo es el plan de salvación que Dios mantuvo oculto desde la eternidad y que reveló y proclamó más tarde ${ }^{21}$.

En él el Apóstol distingue tres fases: el misterio en Dios ${ }^{22}$, el mismisterio revelado ${ }^{23}$ y el misterio proclamado ${ }^{24}$. ¿Y cuál es el contenido de este misterio? San Pablo recurre a diversas fórmulas, que coinciden en el fondo y nos indican que el objeto del misterio es una persona: Cristo ${ }^{25}$.

El término, sin embargo, ha ido evolucionando desde los primeros tiempos y en esa evolución se extiende y desdobla su significado. $\mathrm{Y}$ así llega a significar varias cosas, como el eterno designio de Dios, o plan divino de salvación, la persona misma de Cristo o las prefiguraciones o las profecías. Con Orígenes alcanza el término una nueva perspectiva: la vida de Cristo en la tierra es figura de una realidad celeste, que ha de aparecer en el cielo, o en lo que podemos llamar "nueva tierra" después de los últimos tiempos. El misterio realizado ya en parte en el cristianismo está en plena tensión hacia una consumación en el futuro pareciendo así en todo su relieve el sentido escatológico del mismo. Así encontramos una realidad nueva: la unión entre la realización que tiene ahora su cumplimiento y la esperada. La salvación de Cristo ha de tener su aplicación concreta y temporal ${ }^{26}$.

Más tarde se acentuó ya un nuevo matiz, alcanzando su dimensión a los ritos del bautismo y de la eucaristía, significación que cuajó más

21 De la abundante bibliografía sobre el tema nos limitamos a citar aquí algunos de los ensayos más intemesantes: D. DEBEN, Le mystère paulinien, Eph. Th. Lov. 13 (1936) 405-422; C. SPICQ, Saint Paul. Les Epîtres pastorales., Paris 1948; L. Cerfaux, La Iglesia en San Pablo, Bilbao 1959, 249-262; RigaUX-Grelot, Misterio, V. T B 484-488; H. FRUYTIER, Het word Mystérion in de Catechesen van Cyrillus Jerusalem (Nijmegen 1950); ScHILLEBECKX, De sacramentele heilseconomie (Antwerpen 1952) 21-106; L. BouYER, "Mysterion": Vie Spirituelle Supplement 23 (1952) 397-402; C. MoHRMANN, "Sacramentum dans les plus anciens Textes chrétiens": Harvard Theological Review 47 (1954) $140-152$.

22 I Cor. 2, 7; I Cor. 2, 8; Ef. 3, 9-10; Ef. 1, 10.

23 I Cor. 2,$10 ; E f .3,5 ; E f .3,10 ; E f .3,8,9$.

24 Tit. 1, 1-3; I Tim. 3, 16.

25 Así en Cor. 2, 7-10; Rom. 16, 26; Col. 2, 2-3; Ef. 1, 911; Coll. 27. Es Cristo el que nos merece la participación de los bieneis celestiales y quien la concedie, merecimiento que se extiemde a toda clase de personas, incluso a los gentiles. "En Cristo encarnado y crucificado vemos al misterio divino, oculto y escondido desde la eternidad y que ahora se anuncia y revela en la Iglesia por medio de Cristo (claro está que veladamente), Eph. 1, 4, 9"; ScHMaus, Teología Dogmática VI. Los Sacramentos, 2. edic. Rialp, S. A., Madirid 1963, 22.

26 Smuldres, "La Iglesia como sacramento de salvación": La Iglesia del Vaticano II, Barcelona 1966, 385 ss.; H. U. voN BALTHASAR, "Le mystèrion d'Orgène": Recherches Sc. Relig. 27 (1937) 38-64; ID., "Parole et mystère chez Origène", Paris 1957. 
rápidamente en el término latino que en el griego. Smulders afirma que los dos términos siguieron con parecidas o incluso idénticas significaciones, en un proceso lento y un tanto oscuro, manteniendo la sinonimia desde los siglos iv y v, hasta que en la Edad Media comenzaron a diferenciarse abiertamente ${ }^{27}$.

Por lo que se refiere a San Agustín, el término, en medio de sus fluctuaciones, adquiere carácter propio, que culmina en el doble aspecto de realidad terrena y acción salvífica. Y aunque para Agustín las dos palabras -misterio y sacramento- tengan en un principio el mismo significado, aparte de esas orientaciones en el contenido, se nota ya una tendencia a distinguirlos que tendrá en el futuro una influencia decisiva ${ }^{28}$.

Schmaus confirma esta misma idea cuando afirma que "la teoría de los sacramentos tuvo un impulso decisivo gracias a Agustín, que fue el primero en hacer penetrantes estudios sobre la esencia y significación de los sacramentos. Para ello se sirvió de categorías y representaciones neoplatónicas de forma que el neoplatonismo fue el primer modo de exponer la teología sacramental. Las ideas agustinianas fueron decisivas en lo sucesivo" ${ }^{29}$.

Pero hemos de insistir en que a pesar de los abundantísimos estudios que se han sucedido hasta hoy no se ha llegado todavía a conclusiones definitivas en esta cuestión tan compleja de la sacramentaria agustiniana. "Para San Agustín - continúa Schmaus- el sacrameto es un signo sagrado (signum sacrum); deben distinguirse en el sacramento el signo y el contenido. En los signos no debe mirarse lo que son, sino lo que significan. Para que el signo pueda dar a conocer algo distinto de su ser debe tener cierta semejanza con lo que significa. Portadores de esa semejanza son las cosas naturales y la palabra con que tales cosas naturales son determinadas más concretamente. Cosa natural y palabra fundan el fenómeno visible del sacramento, que por divina virtud se cumple y se hace capaz de producir la realidad sacramental, que es la gracia" ${ }^{30}$.

San Agustín emplea las dos palabras "misterio" y "sacramento"

27 SMULdRES, o. c., 385.

28 T'H. CAMELOT, "Sacramentum. Notes de théologie augustinienne": Revue Thomiste 57 (1957) 429-449.

29 ScHmaus, Teología Dogmática. VI. Los Sacramentios, Ed. Rialp, Madrif $1963,23$.

30 ID., 23-24; J. ENGELS, "La doctrine du signe chez Saint Augustin": Studia Patristica VI, 4, Ed. F. L. Cross, Berlin 1962, 366-372. 
muchas veces con el mismo significado, como puede verse en algunos ejemplos de sus escritos, aun siendo evidente como decimos su tendencra a distinguirlos ${ }^{31}$.

Unas veces habla de los sacramentos como de unos ritos, otras como de unos símbolos y otras como de unos misterios ${ }^{32}$.

En general, y teniendo en cuenta las etapas de la evolución de su doctrina y de su pensamiento, que no permiten una conclusión clara en orden a la definición de sacramento, debe admitirse que para San Agustín el sacramento es un signo de una cosa sagrada, o un signo sagrado, externo y visible, un "signo sensible de la gracia invisible" ${ }^{33}$. Además se trata de un signo divinamente instituído ${ }^{34}$.

El sacramento para San Agustín es algo perceptible por los sentidos, que encierra en sí una realidad, es un signo con un contenido espiritual ${ }^{35}$.

31 "Signum esit enim res, praeter sperciem quam ingerit sensibus, aliud aliquid ex se faciens in cogitationem venire: sicut vestigio viso, transisse animal cujus vestigium est, cogitamus; et fumo viso, ignem subesse cognoscuimus..." (De Doctr. christ. II, 1, 1, PL. 34, 35). "Nimis autem longum est convenienter disputare de varietate signorum, quae cum ad res divinas pertinent, Sacramenta apellantur" (Epist. 138, 1, 7, PL. 33, 527).

32 GrabowsKI cree que los diversos: y abundantes textos en que San Agustín habla de sacramento pudieran agruparse en tres epígrafes: sacramento-rito, sacramento-símbolo y sacramento-misterio. Cfr. GrabowsKi, o. c., 170; C. CouTIER, 0. c., 164-174; F. VAN DER MEER, "Sacramentlum chez Saint Agustin": La Maison Dieu 13 (1948) 50-65; SPALLANZANI, "La nozione di sacramento in Sant'Agostino": Scuola Cattolica serie 6, 9 (1927) 175-88, 258-68.

${ }_{33}$ De doctr. christ. II, 1, 1, PL. 34, 35; De Civ. Dei 10, 5, PL. 41, 282 ; "sacramentum est signum sacrum". Contra Faustum 19, 16, PL. 42, 356-357: "Quid enim aliud quaeque corporalia sacramentia nisi quaedam quasi verba visibilia, sacrosanta quidem...". Quaesti in Hept. III, q. 84. PL. 34, 712. In Joan Evang. tr. 80, 3, PL. 35, 1840: "Detrahe verbum, et quid est aqua nisi aqua? Accedit verbum ad elementum eit fit sacramentum, etiam ipsum tanquam visibile verbum". Epit. a Rom. inchoat. exposi 18, PL. 35, 2101: "ut etiam ipsa sacrosanta signacula, quorum res in eo praeceiserat...". Qudest. in Hept. III, q. 84, PL. 34, 712: "Nam sine ista sanctificatione invisibilis gratiae, visibilia sacra-menta quid prosunt? Merito enim quaeritur, utrum etiam ista invisibilis sanctificatio sine visibilibus sacramentis, quibus visibiliter homo sanctificatur, pariter nihil prositt: quod utioue absurdum est". Contra Faustum 19, 11, PL. 42, 355: "In nullum autem nomen relgionis, seu verum, seu falsum, coagulari homines possunt, nisi aliquo signaculorum vel sacramentorum visibilium consortio conligentur".

34 De doctr. christ. III, 9, 13, PL. 34, 70-71: "Sub signo enim siervit qui operatur aut veneratur alliqum rem significatam, nensciens quid significet: qui vero aut operatur, aut inveneratur utile signum divinitus institutum, cujus vim significationemque intelligit. non hoc veneratur quod videtur et transit, sed illud potius quo talia cuancta referenda sunt".

35 "Para San Agustín los sacramentos no solamente son signos de santificación individual, sino también de unidad religiosa y comunión espiritual. Son lazos visibles que unen a los hombres que se esfuerzan por alcanzar la meta común, si bien contienen también un effecto espiritual, invisible y ultramundano", GRABOWSKI, o. c., 171. 
Es este sentido y estos rasgos peculiares los que interesan para calibrar el contenido de la expresión sacramental de la Iglesia y el alcance de la misma, aun cuando no se la llame expresamente sacramento. Teniendo esto en cuenta hay que admitir que San Agustín apunta ya en su concepción sacramental al doble elemento: realidad terrena y acción salvífica, al sacramento como realidad que produciendo lo que significa y significando lo que produce, es capaz de producir la santificación ${ }^{36}$.

También es digno de notarse el sentido que encierran los signos. Son, según Agustín, "señales dadas" por alguien, lo cual implica que detrás de los signos encontramos a alguien, encontramos a una persona ${ }^{37}$. Un signo es entonces la manifestación de una interioridad, de algo oculto y escondido que se hace palpable a los demás, que se manifiesta con lo que lleva dentro ${ }^{38}$.

\section{Cristo sacramento de Dros. La Iglesia sacramento de Cristo.}

Estas nociones nos hacen ver la enorme dificultad de la problemática sacramentaria en San Agustín y, por tanto, lo difícil que resulta aclarar el concepto sacramental en el pensamiento agustiniano. Pero al mismo tiempo nos sirven para comprender mejor en su dimensión verdadera el alcance de la terminología sacramental aplicada a la Iglesia.

La definición sacramental de la Iglesia, según el sentir de numerosos teólogos que defendieron esta definición y que ahora proclamó el Concilio en algunas fórmulas concretas, da al sacramento el sentido agustiniano de "forma visible de la gracia invisible" 39 . "Sacramento es el eterno designio salvífico de Dios, el cual se revela y realiza poderosamente entre los hombres. O, también, es una acción eficaz de Dios -que comprende tanto la iniciativa y operación divina, como su efecto en el mundo humano- por el cual El, revelando su plan salvífico,

$36 \quad$ Ibid. 171.

37 De Doctr. christ. II, 1, 2, PL. 34, 35-37.

38 Smulders resalta aquí este aspecto de estimable contenido en el pensamiento agustiniano porque implica una invitación personal que de suyo puede dar origen a una comunidad, aunque en la práctica, no siempre llega a conseguirse porque no se acepta la invitación. Por eso al glosar este aspecto de la mentalidad agustiniana comenita: "Las transformaciones que su descripción transitoria intelectualista del signo experimentó, muestran cómo la idea de Agustín fue coartada más y más en un cauce intelectualístico". Cfr. o. c., 389. 39. Denz. n. 876. 
to realiza en esta tierra, para que los hombres reconozcan a su salvador en esa revelación escondida y realización transitoria, crean en $\mathrm{El}$ y lo afirmen en la fe, se dejen poseer por El y en un encuentro personal con єl Redentor, participen de la redención salvación" ${ }^{40}$.

Cristo sacramento de Dios.

En esta perspectiva sacramental hay que encuadrar la terminología de la sacramentalidad aplicada a la Iglesia. Y en este sentido para Agustín Cristo, como Epifanía o manifestación de Dios, en su humanidad, es el sacramento por excelencia. San Agustín llega a afirmar que es "el único sacramento" ${ }^{41}$. Cristo es el sacramento "original" que se convierte un el único camino de salvación.

Cristo es el sacramento de Dios, y lo es por su encarnación. Cristo elevó, y elevándola la divinizó, la naturaleza humana, y la elevó a signo eficaz de la comunicación de Dios al hombre. Jesucristo haciéndose hombre, asumiendo por su encarnación la naturaleza humana, se ha hecho manifestación de la bondad divina y del amor sin límites, el amor inmenso que Dios tiene a los hombres.

De este modo Jesucristo se hace "camino", que conduce a Dios. Viene a unir a Dios con el hombre y al hombre con Dios, para hacer realidad la comunidad de amor entre Dios y el hombre. Esto lo realizó con su vida, muerte y resurrección, que viene a ser la expresión tangible, el signo que aparece ante los hombres y que les descubre a éstos el amor redentor. Es la "señal dada", la llamada. Jesucristo es la realización y manifestación del amor salvífico de Dios para con los hombres, es signo eficaz de la unión íntima del hombre con Dios.

El hombre - como término y destino en Dios- queda así ligado a Cristo de manera que resulta imposible su separación. No es posible separar a Cristo del hombre. Cristo se encarna por el hombre y para el hombre. Su encarnación tiene una finalidad concreta: la redención del hombre, la salvación del hombre. De tal manera que toda la historia se centra en Cristo y El recapitula toda la historia. Antes de la encarna-

40 Cfr. SMuldRes, o. c., 391.

41 Epist. 187, 11, 34, CSEL 57, 113, PL. 33, 845; CoUTURIFR, o. c., 194; Epist. 187, 34, PL. 38, 845. 
ción se orienta ya hacia Cristo y después de la encarnación desemboca en $\mathrm{El}^{42}$. Esto tiene su irealización plena en el Nuevo Testamento.

En el Antiguo Testamento estaba hasta cierto punto como oculta la acción divina. El Antiguo Testamento era como un velo que ocultaba a los hombres ese plan divino y que impedía la visión clara del secreto íntimo de Dios para con la humanidad. Cristo, ahora, en el Nuevo Testamento, haciéndose hombre, rasgará definitivamente ese velo y entonces aparecerá como realidad visible y palpable ese plan misterioso v salvífico de Dios ${ }^{43}$.

\section{J. G. Centeno}

42 Sobre este tema que ha merecido atención eispecial en los últimos años y sobre el que se ha elaborado emsayos de indudable interés puede verse, por ejemplo, J. Danielou, El misterio de la Historia, San Sebasitián 1960; H. URS VON Balthasar, Teología de la Historia, Madrid 1959. Un juicio crítico sobre las diversas tendencias en Z. Alszeghi - M. Flick, "Teologia della Storia": Gregorianum 35 (1954) 256-298.

43 Son ya conocidas las frases agustinianas: "In Vetere Testamento est occultatio Novi, in Novo Testamento est manifestatio Veteris", "In Vetere norum latet, et in Novo vetus patet". De Cathech. rud. 4, 8; Quaest. in Hep 2, 73 PL. 